\title{
Knowledge, attitudes, and perceptions of cupping therapy (CT) in Saudi Arabia-a cross-sectional survey among the Saudi population.
}

\author{
Hanan M. Al-Yousef ${ }^{1 *}$, Syed Wajid ${ }^{2}$, Ibrahim Sales² \\ ${ }^{1}$ Department of Pharmacognosy, College of Pharmacy, King Saud University, Female Campus, Riyadh, Saudi Arabia \\ ${ }^{2}$ Department of Clinical Pharmacy, College of Pharmacy, King Saud University, Riyadh, Saudi Arabia
}

\begin{abstract}
Objective: The aim of this study was to assess the knowledge, attitudes, and perceptions about cupping therapy among the general population of the Kingdom of Saudi Arabia (KSA).

Methods: A descriptive cross sectional online survey was conducted among the general population of KSA. Data was collected by using a structured and self-administered questionnaire. The questionnaire was comprised of 29 questions regarding the knowledge, attitudes, and perceptions about cupping therapy.

Results: A total of 251 of respondents completed the questionnaire. The majority of respondents $(85.5 \%)$ were female and approximately $60 \%$ were using cupping therapy because it was recommended by their physician. More than half of the respondents $(\mathbf{5 4 . 8 \%})$ claimed that they never discuss cupping therapy treatment with their doctors. The majority of participants $\mathbf{7 1 . 5 \% )}$ reported strong trust or belief in cupping therapy. Only $46.4 \%$ of the respondents were aware of situations where cupping is prohibited, most of the participants $(\mathbf{8 9 . 7 \%})$ believed that it is an effective method of treatment, and more than half (58.7\%) think that cupping therapy leads to side effects.

Conclusion: This represents the first description of the knowledge, attitudes, and perception of KSA citizens towards CT. From a governmental perspective, more regulation of cupping and its practitioners is needed. Healthcare providers should be educated regarding cupping and create an open, respectful environment for communication with patients about CT. Patients should likewise consult their physicians about CT use and use caution when selecting CT practitioners.
\end{abstract}

Keywords: Cupping therapy, CAM, Knowledge, Perception, Saudi Arabia.

Accepted on September 18, 2018

\section{Introduction}

Cupping therapy $(\mathrm{CT})$ is an alternative healing method practiced by many cultures particularly Asian and Middle East [1]. It also has gained popularity recently in western countries such as Europe and the United States [2,3]. CT, literally known as sucking, is a process of removing toxins from the blood by placing heated cups on various parts of the body. This allows a local suction to occur which promotes the movement of blood and is believed to ameliorate and/or cure a variety of diseases $[1,4,5]$.

Cupping is subdivided into two further categories: wet or dry cupping. Wet cupping involves the application of cups to areas in which incisions have been made. Dry cupping is performed without any incision [6]. In Muslim and Arab cultures, wet cupping is prodomately used [7]. Previous international reports related the use of cupping therapy to the management of numerous conditions including joint pain, to promote blood circulation, fatigue (extreme tiredness), muscle stiffness [8,9], stroke recovery, elevated blood pressure, musculoskeletal pain, herpes zoster [5,10], and pain relief [11,12] including chronic neck and shoulder pain , and lower back pain [13-17].

In Saudi Arabia (SA) previous studies reported that strong belief in cupping therapy as a modality of treatment for some medical conditions among Saudi population [18]. Similarly another study from Northern border area of SA estimated the general public opinions and attitudes about cupping therapy. Reports revealed positive attitudes towards cupping therapy. In addition, $73 \%$ of participants would recommend cupping therapy to their friends and relatives and 37\% intend to use cupping therapy soon. Interestingly, $75 \%$ of respondent had no experience using cupping therapy [19].

Cupping therapy is considered as a recommended religious practice since 1400 years ago [20]. A review of the literature has indicated that cupping has shown favorable results; however, more rigorous studies are needed. Due to the Islamic prospectious and high interest in cupping specifically in SA, it raises a concern of whether the general public and community in KSA have any basic knowledge and what are their attitudes and perceptions about CT. Previous studies have been limited 
to two regions in SA. Therefore, the objective of this study is to evaluate the knowledge, perceptions and attitudes of general public regarding $\mathrm{CT}$ in different regions of throughout the KSA.

\section{Methods and Materials}

A cross-sectional online survey was conducted during the period from January 2018 to May 2018 targeting in the citizens of KSA. Data was collected by using a standardized questionnaire which was distributed through social networking websites using a chain sampling technique where any participant could recommend additional subjects from among their acquaintances.

The questionnaire was adapted from previous studies published in this regard, collected questionnaires from related studies were redesigned, modified and translated into the Arabic language [21]. The survey questionnaire consisted of a total of twenty-nine questions. The questionnaire included four sections. The first section compiled demographic information. The second section consisted of questions regarding the general attitudes, perceptions, and knowledge regarding CT. The third section dealt with most frequent ailments treated with $\mathrm{CT}$. The fourth section gathered the most common reasons that limit the use of cupping therapy in the Saudi population. The target population for this study were residents of Saudi Arabia aged $15 \mathrm{y}$ and above.

\section{Data analysis}

Descriptive statics including percentages, means, and frequency distribution were calculated for each variable. Statistical Package for Social Sciences version 22.0 (SPSS Inc., Chicago, IL, USA) was used for statistical computations.

\section{Results}

A total of 251 of respondents completed the questionnaire. The overwhelming majority of respondents $(85.5 \%)$ were female. More than half of the respondents were $(68.5 \%)$ residents of the central region of KSA. In addition, most of respondents were aged from $46 \mathrm{y}$ to $60 \mathrm{y}$. The demographic characteristics of the participants are presented in Table 1.

Table 1. Demographic information of respondents from five different regions of Saudi Arabia.

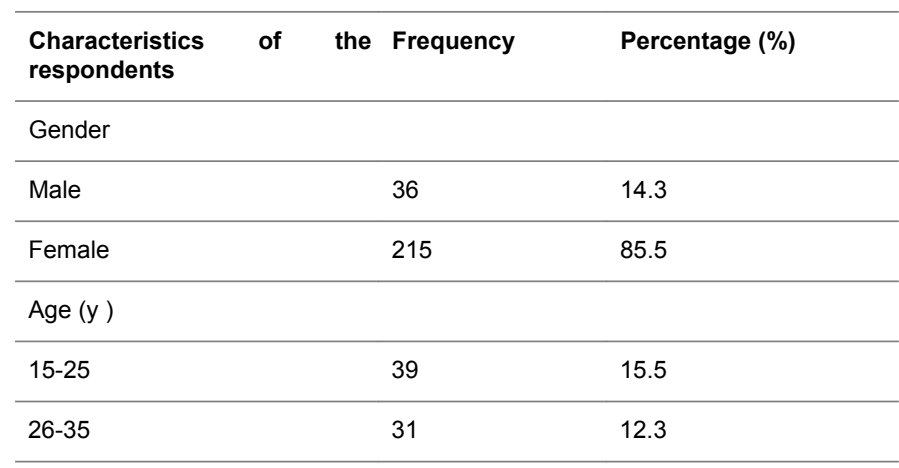

\begin{tabular}{lcc}
\hline $36-45$ & 86 & 34.1 \\
\hline $46-60$ & 95 & 37.7 \\
\hline Marital status & 185 & 73.4 \\
\hline Married & 66 & 26.2 \\
\hline Unmarried & 117 & \\
\hline Employment status & 87 & 46.4 \\
\hline Employed & 47 & 34.5 \\
\hline Unemployed & 18.7 \\
\hline Students & 172 & 15.1 \\
\hline Region & 19 & 7.9 \\
\hline Central & 38 & 0.7 \\
\hline Western & 20 & 7.5 \\
\hline Eastern & 2 & \\
\hline Southern & & \\
\hline North & 17.5 \\
\hline
\end{tabular}

More than half of the respondents $(60.3 \%)$ reported that they were using cupping therapy because their physicians recommended it; however, over half of the respondents (54.8\%) claimed that they never discussed cupping therapy treatment with their physicians. The most common sources of information used for learning more about cupping therapy were books /periodicals $(55.6 \%)$, followed by advice from family and friends $(22.6 \%)$ and social media $(10.7 \%)$ as shown in Table 2 .

The most common reasons for using cupping therapy were treatment $(76.2 \%)$ and recommendations by relatives $(12.3 \%)$. With regards to reliance upon cupping therapy, the study shows that $(71.5 \%)$ of the respondents reported strong trust or trust, while only $28 \%$ of the respondents don't have trust on cupping therapy. Approximately $46 \%$ of the respondents were aware of situations/ailment where cupping is prohibited. Most of the participants $(89.7 \%$ ) reported that CT is an effective method of treatment, and about $60 \%$ of think that cupping therapy leads to side effects as shown in Table 2 .

Table 2. General attitude, perception, and knowledge regarding cupping therapy $(C T)$.

\begin{tabular}{lll}
\hline Cupping therapy & $\begin{array}{l}\text { Frequenc } \\
\mathbf{y}\end{array}$ & $\begin{array}{l}\text { Percentag } \\
\mathbf{e}(\%)\end{array}$ \\
\hline $\begin{array}{l}\text { Has this type of treatment been requested by a } \\
\text { physician? }\end{array}$ & 152 & 60.3 \\
\hline Yes & 99 & 39.3 \\
\hline No & & \\
\hline $\begin{array}{l}\text { Do you inform your doctor about your use of this } \\
\text { treatment? }\end{array}$ & 113 & 44.8 \\
\hline Yes & 138 & 54.8 \\
\hline No & & \\
\hline
\end{tabular}


Knowledge, attitudes, and perceptions of cupping therapy (CT) in Saudi Arabia-a cross-sectional survey among the Saudi population

\begin{tabular}{|c|c|c|}
\hline \multirow{2}{*}{\multicolumn{3}{|c|}{$\begin{array}{l}\text { What are your sources for using } \\
\text { treatment? } \\
\text { Internet (social media) }\end{array}$}} \\
\hline & & \\
\hline Media (T.V. ,Podcasting, Magazines, etc ) & 27 & 10.7 \\
\hline Books/Periodicals & 27 & 10.7 \\
\hline \multirow[t]{2}{*}{ By family and friends used } & 140 & 55.6 \\
\hline & 57 & 22.6 \\
\hline \multicolumn{3}{|l|}{ Common practicing places for $\mathrm{CT}$} \\
\hline Contact a specialist at home & 139 & 55.2 \\
\hline Clinical centers/hospitals & 37 & 14.7 \\
\hline Popular therapists & 75 & 29.8 \\
\hline \multicolumn{3}{|l|}{ What are the reasons for using CT? } \\
\hline Prophylactic & 28 & 11.1 \\
\hline Treatment & 192 & 76.2 \\
\hline Recommended by relatives & 31 & 12.3 \\
\hline \multicolumn{3}{|c|}{$\begin{array}{l}\text { Do you trust CT as an effective alternative } \\
\text { treatment? }\end{array}$} \\
\hline Strong trust & 141 & 56 \\
\hline Trust & 39 & 15.5 \\
\hline No trust & 71 & 28.2 \\
\hline \multicolumn{3}{|c|}{$\begin{array}{l}\text { Are you aware of situations where cupping is } \\
\text { prohibited? }\end{array}$} \\
\hline Yes & 117 & 46.4 \\
\hline No & 134 & 53.2 \\
\hline \multicolumn{3}{|c|}{ Have you noticed any effectiveness by using CT? } \\
\hline Yes & 226 & 89.7 \\
\hline No & 25 & 9.9 \\
\hline \multicolumn{3}{|l|}{ Do you think CT have side effects? } \\
\hline Yes & 148 & 58.7 \\
\hline No & 103 & 40.9 \\
\hline \multicolumn{3}{|c|}{$\begin{array}{l}\text { Have you noticed the prevalence of CT in your } \\
\text { region? }\end{array}$} \\
\hline Yes & 94 & 37.3 \\
\hline
\end{tabular}

\begin{tabular}{lcc}
\hline No & 157 & 62.3 \\
\hline Do you urge people to do CT in your area? & & \\
\hline Yes & 238 & 94.4 \\
\hline No & 13 & 5.2 \\
\hline Do you think how old is appropriate for cupping? & & \\
\hline Childhood & 2 & 0.8 \\
\hline Adults & 142 & 56.3 \\
\hline Elderly & 37 & 14.7 \\
\hline I don't know & 70 & 27.8 \\
\hline
\end{tabular}

Table 3 details the common diseases reported by study subjects to be treated with cupping therapy. The most frequent ailments are shoulder/back pain $(51.7 \%)$, blood circulation stimulation (43.8\%), headaches (31\%), reliving joint pain (30.6\%), dysmenorrhea $(15.1 \%)$ relief/treatment, to control or reduce hypertension $(7.5 \%)$, and to relieve/treat sciatica $(7.1 \%)$.

According to the study results, the most reasons that limit using cupping therapy are the scarcity of CT centers $(60.5 \%)$, and questioning appropriate cleaning of tools and/or the inability to provide specialists (34.6\%) (Table 4).

Table 3. Common ailments by using cupping therapy and common reasons that prohibit the uses of $C T$.

\begin{tabular}{lll}
\hline Questions & Frequency & Percentage (\%) \\
\hline What does cupping therapy treat? & & \\
\hline Relieve joint pain & 77 & 30.6 \\
\hline Relieve shoulders back pain & 130 & 51.7 \\
\hline Relieve sciatica & 18 & 7.1 \\
\hline Decrease HTN & 19 & 7.5 \\
\hline Relieve headache & 78 & 31 \\
\hline Relieve dysmenorrhea & 38 & 15.1 \\
\hline Relieve varicose veins & 5 & 1.9 \\
\hline Decrease HDs & 8 & 3.1 \\
\hline Stimulate circulation & 110 & 43.8 \\
\hline
\end{tabular}

Table 4. Most common reasons preventing to do cupping therapy.

\section{Scenarios}

Frequency

$\%$

Which of the following reasons will prevent you from using CT?

\begin{tabular}{lcc}
\hline Few CT centers in my region & 60.5 & 152 \\
\hline Doubts regarding appropriate cleaning of tools and/or the inability to provide specialists & 34.6 & 87 \\
\hline I have diseases that prevent me from practicing cupping & 4.7 & 12 \\
\hline
\end{tabular}




\section{Discussion}

The results of this study represent the first publication describing the knowledge, attitudes, and perceptions of cupping therapy on a nationwide scale in KSA. As opposed to other cultures and societies worldwide, the belief and trust in cupping therapy in KSA is founded upon prophetic advice based upon revelation as opposed to cultural traditions exclusively [22]. Furthermore, this explains the high prevalence of cupping therapy in KSA among residents from $4-45 \%$ up to $92 \%[18,23]$.

Although only $44.8 \%$ of study participants have informed their physicians of their use of CT, over $60 \%$ reported that cupping therapy had been recommended by their physicians. Albalawi reported that only $6.5 \%$ of participants would recommend cupping therapy without medical advice in Tabuk, SA [18]. However, this conflicts previous reports from Al-Rowais et al. who reported that only $14.2 \%$ of healthcare practitioners in SA refer their patients to complementary and alternative medicine practitioners [23].

Under half (46.4\%) were aware of situations where cupping is prohibited. This differs from published data from Tabuk in which only $18.5 \%$ knew that cupping is contraindicated in certain disease states [18]. However, Alshamri et al. reported that $59 \%$ of participants responded that cupping is contraindicated in these situations [19]. It is not surprising since over $75 \%$ of their study population had obtained beyond a university educational degree.

Respondents indicated that $55.2 \%$ currently contact a specialist to visit their home for CT, and only $14.7 \%$ utilize clinical centers and/or hospitals. Furthermore, hindrances to therapy include a fear of contaminated equipment. Both Albalawi et al. and Mujtaba reported that participants encouraged CT to be performed in specialized centers, $49 \%$ and $96 \%$, respectively $[18,19]$. Moreover, $46.5 \%$ knew that certain diseases can be transmitted by contaminated equipment [18].

This study has limitations. The majority of participants were from the central region of KSA; therefore, the study may not truly represent the cultural differences in other areas of the country. In addition, further demographic data, such as educational status, may be helpful in providing a clearer description of the influences upon participant use of CT.

\section{Conclusion}

This represents the first description of the knowledge, attitudes, and perception of SA citizens towards CT. From a governmental perspective, more regulation of cupping and its practitioners is needed. Healthcare providers should be educated regarding cupping and create an open, respectful environment for communication with patients about CT. Patients should likewise consult their physicians about CT use and use caution when selecting CT practitioners. Future research is needed to further describe the opinions regarding $\mathrm{CT}$ in KSA as well as more clinical research to support its therapeutic benefits.

\section{Acknowledgment}

This research project was supported by a grant from the "Research Center of the Center for Female Scientific and Medical Colleges", Deanship of Scientific Research, King Saud University.

\section{References}

1. What is cupping therapy? http: // www.britishcuppingsociety.org/.

2. Farhadi K, Schwebel DC, Saeb M, Choubsaz M, Mohammadi R, Ahmadi A. The effectiveness of wetcupping for nonspecific low back pain in Iran: a randomized controlled trial. Complement Ther Med 2009; 17: 9-15.

3. The benefits of cupping. Available from: URL: http: //thebenefits.blogspot.com/2018/04/thebenefits-ofcupping.html.

4. Arab news. MoH sets guidelines for cupping therapy. Availbe at http: //www.arabnews.com/saudi-arabia/news/ 712891 last accessed at April 162018.

5. Cao $\mathrm{H}$, Han $\mathrm{M}, \mathrm{Li} \mathrm{X}$. Clinical research evidence of cupping therapy in China: a systematic literature review. BMC Compl Alt Med 2010; 10: 70.

6. Dnyaneshwar KJ. Cupping therapy: an ancient alternative medicine. J Phy Fit Treatment Sports 2018; 3.

7. AlBedah A, Khalil M, Elolemy A, Elsubai I, Khalil A. Hijama (cupping): a review of the evidence. Focus Alt Compl Ther 2011; 16: 12-16.

8. Cao H, Hu H, Colagiuri B, Liu J. Medicinal cupping therapy in 30 patients with fibromyalgia: a case series observation. Forschende Komplementärmedizin 2011; 18: 122-126.

9. Lin $\mathrm{KC}$, Chen ML, Yeh ML, Hsu CH, Chen YL, Chou P. Prevalence, pattern, and predictors of use of complementary and alternative medicine in Taiwan. Taiwan J Publ Health 2009; 28: 53-68.

10. Lee MS, Kim JI, Ernst E. Is cupping an effective treatment? An overview of systematic reviews. J Acupunct Meridian Stud 2011; 4: 1-4.

11. Kim SB, Lee YH. Numerical analysis of the change in skin color due to ecchymosis and petechiae generated by cupping: a pilot study. J Acup Merid Studies 2014; 7: 306-317.

12. Huang S, Cao Y. Cupping therapy. J Chinese Med 2006; 82: $52-57$.

13. Kim TH, Kang JW, Kim KH. Cupping for treating neck pain in video display terminal (VDT) users: a randomized controlled pilot trial. J Occup Health 2012; 54: 416-426.

14. Lauche R, Cramer H, Hohmann C. The effect of traditional cupping on pain and mechanical thresholds in patients with chronic nonspecific neck pain: a randomised controlled pilot study. Evid Based Compl Alt Med 2012; 2012: 10 .

15. Yuan QL, Guo TM, Liu L, Sun F, Zhang YG. Traditional Chinese medicine for neck pain and low back pain: a 
Knowledge, attitudes, and perceptions of cupping therapy (CT) in Saudi Arabia-a cross-sectional survey among the Saudi population

systematic review and meta-analysis. PLoS One 2015; 10 : 0117146.

16. Tamer S. Cupping therapy is beneficial for treating numerous diseases. Available from: http: // www.naturalnews.com/022727_cupping_therapy.html

17. The benefits of cupping. Available from: URL: http: // thebenefits.blogspot.com/2013/04/the-benefits-ofcupping.html

18. Amal MAB, Abdulrahman HA, Azza OA, Tarig HM. Public perceptions of cupping therapy in Tabuk city, Saudi Arabia. Int J Med Sci Publ Health 2016; 5.

19. Amir MA, Ali M. The opinion and attitude about cupping therapy among general population of Northern region of Saudi Arabia. EJPMR 2017; 4: 68-73.

20. Qureshi NA, Shaban T, Elsubai IS, Alqaed MA, Gazzaffi I, El-Olemy AT, Al-Bedah AM. History of cupping (Hijama): a narrative review of literature. J Integr Med 2017; 15: 172-181.

21. Ahmad HAG, Hanan MA, Fatema A, Alla H. Beliefs and attitudes of para-medical college staff towards complementary and alternative medicine. Afr J Trad 2016; 13: $170-177$.

22. El Sayed SM, Baghdadi H, Abou-Taleb A, Mahmoud HS, Maria RA, Ahmed NS, Helmy Nabo MM. Al-hijamah and oral honey for treating thalassemia, conditions of iron overload, and hyperferremia: toward improving the therapeutic outcomes. J Blood Med 2014; 5: 219-237.

23. Alrowais NA, Alyousefi NA. The prevalence extent of complementary and alternative medicine (CAM) use among Saudis. Saudi Pharm J 2017; 25: 306-318.

\section{*Correspondence to}

Syed Wajid

Department of Clinical Pharmacy

College of Pharmacy

King Saud University

Saudi Arabia 